\title{
Recording and analyzing eye-position data using a microcomputer workstation
}

\author{
C. F. NODINE, H. L. KUNDEL, L. C. TOTO, and E. A. KRUPINSKI \\ University of Pennsylvania, Philadelphia, Pennsylvania
}

\begin{abstract}
This paper presents a PC-based eye-position data collection and analysis system. Software routines are described that supplement hardware calibration procedures, improving data-collection accuracy and reducing the number of unusable trials. Collected eye-position data can be remapped over a displayed stimulus image and spatially and temporally represented by parameters such as individual fixations, clusters of fixations (Nodine, Carmody, \& Kundel, 1978), cumulative clusters, and gaze durations. An important feature of the system is that the software routines preserve scan-path information that provides a sequential dimension to the analysis of eye-position data. A "hotspot" analysis is also described, which cumulates, across 1 or more observers, the frequency of eye-position landings or "hits" on designated areas of interest for a given stimulus. Experimental applications in the fields of radiology, psychology, and art are provided, illustrating how eye-position data can be interpreted both in signal detection and in information-processing frameworks using the present methods of analysis.
\end{abstract}

Eye-position recordings provide an important source of data for making inferences about relationships between scanning, fixating, and interpreting pictures and text. Like other researchers (e.g., Cornsweet, 1970), we use the term eye-position recording rather than eye-movement recording, because our analyses primarily focus on the grouping of landing positions of the eye (i.e., fixations and fixation clusters) relative to information displayed in a picture, rather than on the saccades that describe the movements between landings. Thus, in addition to traditional analyses of the distributions, durations, and scan paths, the locus of clusters of fixations and associated gaze-duration dwell times is correlated with specific targets in a given picture.

The eye-position recording system described here was originally developed to study how expert interpreters of medical images (typically radiologists) scan X-ray pictures of human anatomy for abnormalities, and what kinds of features on the X-ray pictures influence scanning strategies during visual search and target detection. An earlier version of the eye-position system has been described by Carmody, Kundel, and Nodine (1980). However, recent changes in microprocessor and display performance have been introduced that improve data acquisition and analysis, increasing the scope of the quantitative analysis of eye-position data. We have taken advantage of this new technology in redesigning our original system, and we think that these developments may be of interest to other

This research was supported in part by NIH Research Grant CA32870, from the National Cancer Institute, USPHS, DHHS. Requests for reprints and software programs should be addressed to C. F. Nodine, Pendergrass Laboratory, 308 Stemmler Hall, University of Pennsylvania, Philadelphia, PA 19104-6086. researchers who use pictorial displays in their work on problems of visual search and detection of targets.

The new system has a number of advantages over the old one. First, an (ISA/EISA bus) 80286/386 microprocessor can be used as the host processor, making the eye-position monitoring system accessible to a broad range of computers. Second, the fixation and cluster algorithms that we use to study visual search and diagnostic radiographic interpretation are generalizable to many visual tasks that require the interpretation of pictorial information. In particular, the quantitative analyses of eye position are specifically designed to provide data that are useful for modeling visual search, detection, and interpretation of objects in pictorial scenes within an informationprocessing framework. Finally, the data-collection and analysis algorithms are independent of the display resolution. We have used the algorithms with both lowresolution $(512 \times 512)$ and high-resolution $(2,048 \times$ $2,048)$ display monitors.

This paper is divided into three sections. The first deals with the recording of eye-position data and includes general descriptions of hardware and software calibration and data-collection procedures. The second section describes the analysis of eye-position data-the sequential formation of fixations, the clustering of these fixations, and the spatial mapping of these measures over the displayed image. The final section illustrates how spatially coded eyefixation data are used to analyze several different visual information-processing tasks.

\section{RECORDING OF EYE POSITION}

\section{System Hardware}

Our system uses a Virtual Imaging 2000 (Virtual Imaging, Sunnyvale, CA) workstation that is capable of dis- 
playing $1,024 \times 1,024 \times 8$ bit images on 17 -in. blackand-white monitors. Sixteen megabytes of image RAM are accessible to software, enabling rapid display updates during series of trials. The VI 2000 is driven by a host 80286 (IBM-PC-compatible) microprocessor with an EGA adapter driving the experimenter's console. All software is written in Microsoft C, version 5.0. The latest versions of the programs described here are available on request from the authors. The strength of our system lies in the software techniques used to collect, calibrate, and analyze the eye-position data. Adapting the software to other display hardware will require modifications of the display-related commands, but the data-collection, calibration, and analysis algorithms are completely generalizable to other systems.

Eye position is monitored by an Eye-Trac Model 210 eye movement monitor (Applied Science Laboratories, Waltham, MA), which measures eye position by using pulsed infrared light. A sensing device, consisting of an infrared light-emitting diode and two phototransistor detectors, is mounted on eyeglass frames and positioned in front of each eye. The $x$-coordinate (horizontal) data are sampled from the right eye, and $y$-coordinate (vertical) data are sampled from the left eye. The infrared light is reflected from the cornea back to the set of detectors, generating voltages that can be spatially mapped to indicate positions of gaze over the display field. The digital output of the monitor is sampled via an OmniComp AT11 8-bit digital interface board (OmniComp, Houston, TX).

A mouse is also linked to the workstation, and its pushbutton is used in the calibration procedure (described below). The mouse can also be used to control the movement of a cursor on the monitor and to localize targets during search.

\section{Calibration Procedures}

The Eye-Trac monitor allows for calibration through electronic adjustment of offset, gain, linearity, and crosstalk compensation using potentiometers. However, the calibration process can be simplified by using software routines (following gross manual adjustments) to correct for residual crosstalk and nonlinear response of the EyeTrac monitor. After the initial manual adjustments have been made, the observer fixates each dot on a 9-dot $(3 \times 3)$ calibration pattern, and pushes a button while fixating the center of each dot. These data are then used to generate a pair of mapping equations (second-degree polynomials) that transform the raw eye-position data to spatially corrected data within the display space. Correction is especially useful when gross manual adjustments are unable adequately to eliminate nonlinearities or channel crosstalk in the calibration data.

The spatially corrected calibration pattern can be evaluated by the software before the stimulus image is presented. If the calibration is unacceptable (i.e., there is a large disparity between the $3 \times 3$ calibration pattern and the eye-position pattern), the recording sensors can be repositioned, the gain adjusted, or the bias in linearity or crosstalk changed, and the calibration procedure can be repeated. Further gross adjustments are usually unnecessary after an initial calibration procedure, although occasional fine-tuning may be necessary. Fixation of the 9-dot pretrial calibration pattern is repeated before every trial. Each calibration dot subtends $0.25^{\circ}$ of visual angle from a viewing distance of $70 \mathrm{~cm}$, and the entire $3 \times 3$ pattern subtends approximately $20^{\circ}$ of the $24^{\circ}$ field. After each data-collection trial (usually 10-30 sec), the observer fixates a 5-dot posttrial calibration pattern.

\section{Calibration is Necessary to Measure Accuracy}

Accuracy, a measure of the system's ability to locate correctly the position of gaze, is primarily dependent on the observer's ability to maintain a fixed head position. The quality of the eye-position data for a given trial can be expressed quantitatively by measuring the goodness of fit of all the spatially corrected calibration eye-position data both to the $3 \times 3$ pretrial calibration pattern and the 5-dot posttrial calibration pattern (14 data points). Taking into account all 14 data points reflects two aspects of accuracy: how close the locus of gaze is to the actual calibration dots, and how reliably the observer refixates a sample of 5 of these dots after viewing a test image. Both aspects of accuracy are important, and we have chosen to combine these two aspects into an overall measure of accuracy.

Our measure of accuracy is derived by calculating the mean distance between the actual calibration positions and the corrected eye-position calibration data. The median of this measure is $0.31^{\circ}$, as obtained by 4 observers, each carrying out 10 calibration trials. The frequency distribution in Figure 1 shows that, approximately $80 \%$ of the time, the average distance data fall below $0.6^{\circ}$. The mode of this distribution is $0.27^{\circ}$. These data demonstrate that on a significant number of trials, accuracy, as defined by the average distance between the calibration dots and their associated eye-position data, is approximately $0.3^{\circ}$.

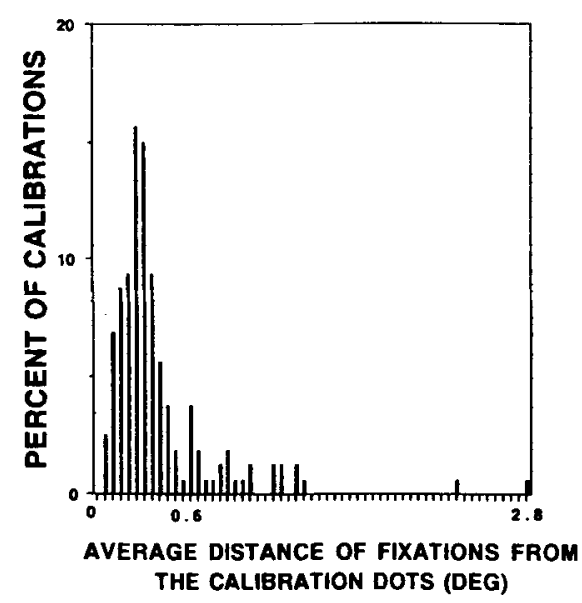

Figure 1. Average distance of fixations from their corresponding displayed calibration points shown as percentage of total calibration trials $(n=4 \times 14 \times 40=2,240)$. Over $80 \%$ of the fixations were within $0.6^{\circ}$ of the displayed calibration points. 
An excellent calibration does not preclude the possibility that head movement occurred during the trial, and that the head returned to its pretrial position prior to postcalibration. However, our experience both in monitoring head movements and in observing changes in eye-position data due to deliberate shifts in head movement indicates that this rarely occurs. Since the primary cause of head movement is fatigue, muscle strain, or gravity, the head rarely returns to its original position once it has moved. The effect of gross head movements is evidenced by a linear shift in the postcalibration pattern, which is easily detected when the 5-dot posttrial calibration data are compared with the data from the corresponding 5 pretrial calibration dots. When this occurs, we stop, give the observer a short rest, and then resume data collection.

Although a bite board may increase accuracy (Mackworth, 1976), we think that an accuracy of $\leq 0.6^{\circ}$ is acceptable to identify and correlate eye position with the spatial locations of most objects in pictorial scenes. When the images that we employ are displayed, they subtend about $22^{\circ}$ of visual angle. We chose a combination headand chinrest, which is a less constraining method of limiting head movement, because the time that our observers can spend being tested is limited, and we thought that the simplicity and comfort of using a chinrest outweighed the gain in accuracy that might be achieved with a bite board.

\section{EYE-POSITION DATA COLLECTION AND ANALYSIS}

\section{Eye-Position Data Collection}

A typical 20-sec data-collection trial yields $1,040 x, y$ coordinate pairs of eye-position data, which are stored on disk as an ASCII file. Each file consists of a $2 \mathrm{~K}$-byte header containing information about the experiment's name, trial number, date, image viewed, observer name, calibration quality, observer decision, confidence, and other pertinent details. The header is followed by the uncorrected calibration data and the spatially corrected $x, y$ coordinate data pairs representing the position of gaze during the trial in display-screen coordinates.

The $x, y$ coordinate pairs of eye-position data are collected during stimulus presentation at a rate of 832 pairs per second. The EGA adapter vertical retrace status bit, which is accessible to the software, provides a convenient timing pulse that paces the data sampling. These samples are grouped into aggregates of 16 coordinate pairs and are averaged, yielding $52 x, y$ "raw" data pairs per second. The raw data are stored in data arrays for subsequent spatial correction. Spatial correction of the raw data is accomplished by remapping the data using the polynomial equations generated by the pretrial calibration procedure. The spatially corrected data are stored on disk and used in all subsequent data-analysis programs.

\section{Eye-Position Data Analysis}

The computer algorithms and thresholds described below were chosen primarily to investigate how radiologists search chest $\mathrm{X}$-ray images for lesions embedded in the lungs. We are particularly interested in using the radiologists' scanning strategies to indicate where fixations tend to group, or cluster, on the image. We believe that these fixation groupings, or "clusters" of fixations, identify areas of perceptual interest and indicate how much time is spent in visually scrutinizing objects in each of these areas. The clustering of fixations around an object of interest is measured by gaze duration, which indicates the depth of visual information processing. We are also interested in how the locations of clusters and their associated gaze durations relate to diagnostically significant objects in X-ray pictures. The algorithms described are, however, generalizable to many visual-search, detection, and picture-interpretation tasks (see Applications) in which eye-position data are used to make inferences about what parts of a picture are being processed by foveal vision.

The formation of both fixations and clusters is based on the determination of whether or not successive data points exceed certain spatial and temporal thresholds (see Figure 2). Thus, the exact spatial and temporal sequence in which the eye samples the image during a trial is a crucial factor that is incorporated into our fixation and clustering algorithms. Our method of defining fixations and clusters by taking into account the scan path is uniquely different from most alternative clustering approaches in which fixation data are grouped on the basis of the density of eye-position data at various spatial locations on the image without regard to scan-path information (see, e.g., Latimer, 1988, and Scinto \& Barnette, 1986, for different approaches).

Scan paths are constructed by connecting sequential fixations or clusters with lines. The direction of movement is from a previous cluster or fixation to a more recent cluster or fixation. Without scan path information, it is impossible to distinguish a single extended gaze from multiple shorter gazes formed by nonconsecutive refixations of a given display location. We analyze eye-fixation patterns with the scan path factored into the analysis, since signal detection questions are often linked to a suspected target's being fixated and subsequently, but not necessarily sequentially, refixated for verification.

By noting the temporal order in which fixation clusters are generated, and by measuring the length of the scan paths connecting the clusters, we have also been able to examine search strategies. Several studies have shown that differences in scanning strategies are related to the expertise of the observer (Gale \& Worthington, 1983; Kundel \& LaFollette, 1972). These differences in scanning strategy have generally been attributed to changes in cognitive schema brought about by medical training and experience (Gale \& Worthington, 1984; Lesgold, Rubinson, Feltovich, Glaser, Klopfer, \& Wang, 1988). Thus, scan-path information may contain a valuable dimension for characterizing search strategies, and particularly for characterizing search efficiency.

One way to characterize search strategies is to analyze the scan-path length of a series of fixation clusters gener- 


\section{Eye Position Grouping Algorithm Flow Chart}

$d=0.5 \mathrm{deg}$ for fixations

$d=2.5 \mathrm{deg}$ for clusters

Initialize Running Mean

to first $x, y$ pair

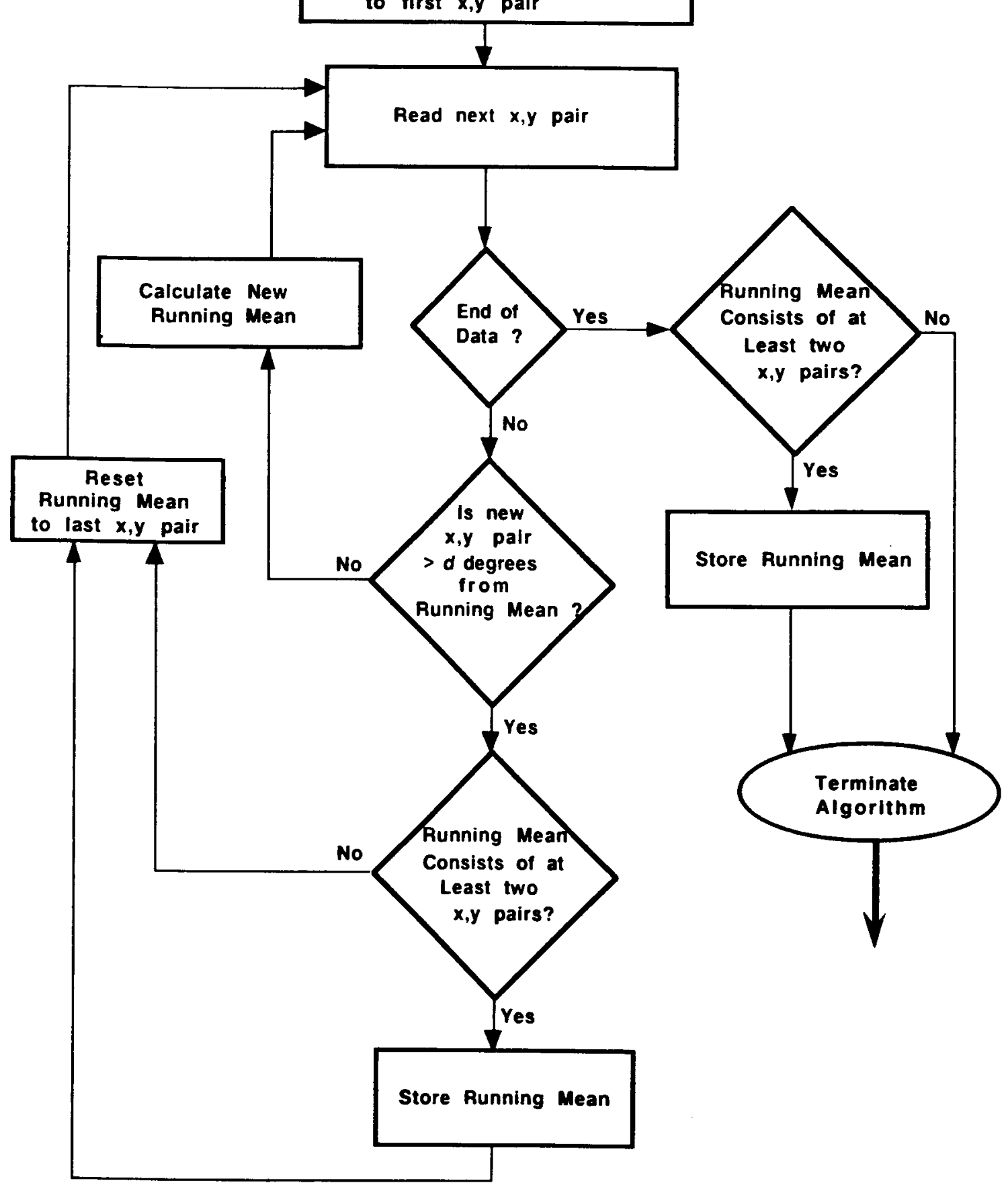

Figure 2. Flowchart of the rules used for grouping raw data into fixations and fixations into clusters. The spatial and temporal threshold values can be varied, depending on the nature of the search task and the target. 


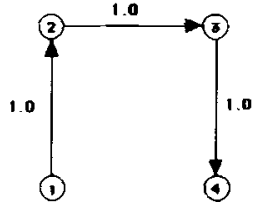

(a) TOTAL $=3.0$

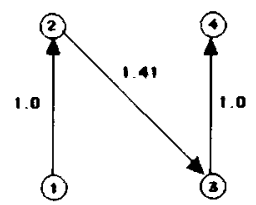

(b) TOTAL $=3.41$

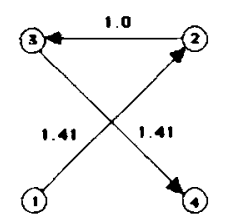

(c) TOTAL $=3.02$
Figure 3. An analysis of search efficiency as measured by calculating the total scan-path length connecting four fixation clusters. Each pattern is associated with only three scan-path segments, but the connecting order and direction differ. The four fixation clusters are represented by circles; the numbers within the circles indicate the temporal order in which they were generated. The lines connecting the circles show the scan path. The length of the lines connecting fixations reflects distance between clusters. Viewing order in the three illustrations differs, and this affects path length or total distance as indicated by the total values, which may be used as a measure of search efficiency.

ated during a visual-search task. For example, Figure 3 shows the spatial locations of four fixation clusters (represented by circles). The connecting lines, which indicate the scan paths, show three ways in which the four fixation clusters could have been generated temporally. Note that the four fixation clusters are connected in each case by only three scan-path segments, but that the order, direction, and total lengths are quite different. These different lengths may be useful in differentiating the efficiency of the different search strategies (see Stark \& Ellis, 1981, pp. 214-215).

A fixation is defined by rules for the spatial and temporal grouping of $x, y$ data pairs. We use a running-mean calculation to determine the spatial location of groupings of data points that ultimately become fixations. In the grouping operation, each successive data point is compared with the mean of the preceding point or set of points. If the new data point is less than a given spatial threshold angle from the mean, it is considered a part of the forming fixation. Typically, $0.5^{\circ}$ is used as the spatial threshold, although the algorithm allows the threshold to be set to any value, depending on the task and analysis requirements. Since the grouping algorithm uses a running-mean calculation, the mean of a forming fixation is recalculated as each new (below threshold) data point is added. This recalculated mean then becomes the reference point for determining whether or not a subsequent data point passes the spatial threshold. Successive data points contribute to the mean of the forming fixation unless the threshold angle is exceeded, which signals the end of the current fixation. The mean is used as a reference point for connecting scan-path lines.

In conjunction with the spatial threshold, a temporal threshold is also applied to the data to define the minimum duration for a forming fixation. Again, the algorithm allows for different settings of the temporal threshold, depending on the task and analysis requirements. If the minimum number of necessary data points chosen to define a forming fixation is 2 , the minimum duration of a fixation is $38 \mathrm{msec}$, which represents the elapsed time of
2 clock cycles of 19 msec each in our computer, running at $52 \mathrm{~Hz}$. A minimum fixation duration of $38-95 \mathrm{msec}$ (2-5 data points) is typically chosen in our visual-search studies. The choice of temporal threshold depends on how accurately the experimenter wishes to identify the spatial location of landing positions without also including a part of the saccade, since the sampling rate is high enough to catch long saccades "on the fly."

Fixations are grouped into clusters by using runningmean calculations that are nearly identical to those used for the formation of fixations from eye-position data, except for the thresholds chosen. The minimum number of fixations per cluster is usually set to 2 , to differentiate them from single, isolated fixations. Single, isolated fixations generally correspond to places where the eye briefly lands after a long saccade. Corrective saccades usually follow as the observer focuses in on the feature of interest at which the saccade was aimed. The cluster distance threshold is typically set at $2.5^{\circ}$. When this value is used, a fixation that falls $2.6^{\circ}$ away from the mean of the group of preceding fixations marks the end of the forming cluster and the possible beginning of a new fixation cluster.

\section{Analysis Playback}

After a trial is complete and the data are stored, the eye-position pattern can be superimposed over the stimulus image and played back in real time. The playback routine contains a menu that allows the experimenter to choose which option or combination of options he/she wishes to display. Figure 4 shows four playback options: (a) eye-position data, (b) fixations, (c) clusters, and (d) fixations and clusters with scan path.

The playback analysis of scan paths formed by connecting the centers of sequential fixations or clusters (i.e., means) by lines reveals the order in which elements in the stimulus are fixated. The scan path has been used to infer the sequence in which pictorial elements are encoded into visual memory (Locher \& Nodine, 1974; Noton \& Stark, 1971). Scan-path records also provide a highly informative visual representation of the extent to which a viewer distributes his/her gaze over a stimulus; and in a search task, they illustrate the type of search strategy employed. Analysis of the scan path can be done to determine whether the general scanning pattern is random or follows some predetermined course (see, e.g., Kundel \& LaFollette, 1972; Kundel, Nodine, Thickman, \& Toto, 1987; Kundel \& Wright, 1969).

In the data-analysis playback mode, circles are drawn around cluster locations. The size (radius) of each circle reflects the spatial dispersion of the contributing fixation data. The size of the cluster can vary, because the clustering algorithm uses a running-mean calculation. Thus, a cluster with contributing fixations that were never more than $1.0^{\circ}$ from the running mean will have a tighter spatial dispersion (and smaller playback circle) than will a cluster with contributing fixations that generally fell $2.4^{\circ}$ from the running mean, yielding a greater spatial dispersion and a larger playback circle. 

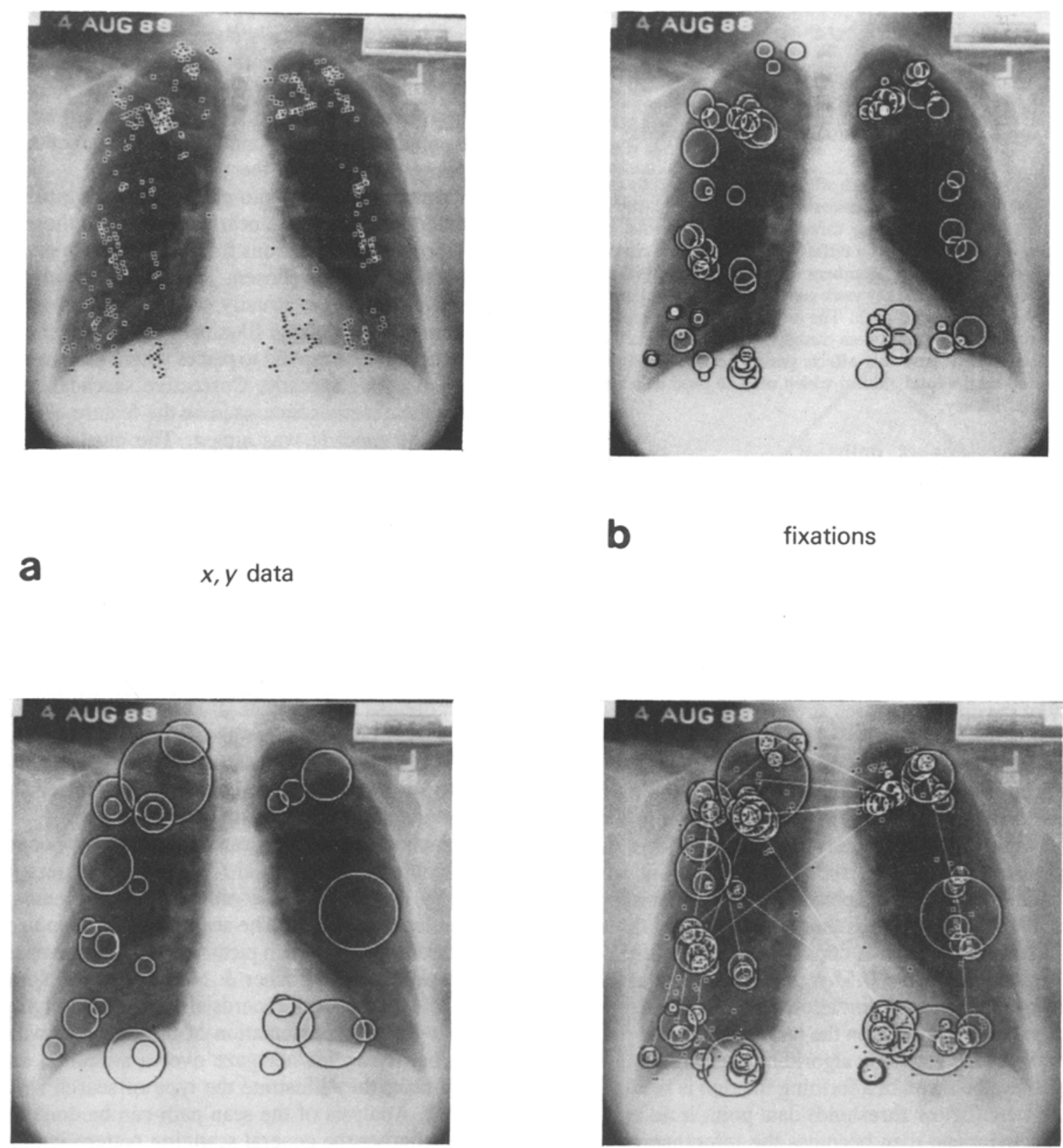
C
clusters

d

comb

Figure 4. Four levels of eye-position data analysis: (a) Small squares are the spatially corrected $x, y$ data (52 samples/sec). (b) Small circles are fixations produced by grouping the $x, y$ data using a spatial threshold of $0.5^{\circ}$ and a temporal threshold of 33 msec. (c) Large circles are clusters of fixations produced by grouping fixations by using a spatial threshold of $2.5^{\circ}$ and a temporal threshold of 66 msec; cluster circle sizes reflect a measure of the dispersion of contributing data. (d) Combination of the three levels above, plus scan path; lines connect centroids of clusters. 
An important post hoc analysis of the data, which does not rely on scan-path information, can be done after a trial. This analysis determines whether any clusters are spatially coincident with any other clusters generated during a given trial. We consider cluster spatial coincidence as consisting of clusters closer than $2.5^{\circ}$ (center-to-center). These coincident clusters indicate locations on the image that were fixated and refixated after there were intervening fixations on one or more spatially distinct image locations. Since the scan path is preserved in the original data collection and storage, we can determine how many times an object is fixated and refixated, and how many noncoincident clusters intervene between coincident clusters. Nonconsecutive refixations of an object may still signal a visual comparison strategy.

By aggregating the durations of fixations within spatially coincident clusters, we can obtain a measure of the cumulative time spent gazing at a fixation-defined area of interest on the image that is not sequentially constrained. The cumulative time measure on each of these circumscribed areas, referred to as gaze duration (see Just \& Carpenter, 1988), is assumed to be a reflection of depth of visual information processing.

\section{Image Manipulation}

The VI 2000 system has a number of imagemanipulation functions that can be useful in various experimental tasks. Some of the image-manipulation techniques used in our radiological studies include zoom, rove, and linear and nonlinear intensity transformations. Since many of our studies necessitate knowing the precise location of search targets, we digitally simulate the tumor targets in chest radiographs and correlate fixation/gazeduration data with known image features by using the center of the fixation cluster to define a circle representing the target zone for identifying a "hit." Typically, the radius of the target zone is $2.5^{\circ}$. The rationale for using this value is described below. We interpret the results using receiver operating characteristic (ROC) analysis.

It is also possible to process the images before importing them into the system. For example, we have conducted experiments on the visual exploration of works of art. One variable thought to influence such exploration is the level of symmetry, or balance, that influences the design of visual compositions (Berlyne, 1971). We have taken various works of art and deliberately modified the arrangement of pictorial elements to affect overall compositional balance (Nodine, Locher, \& Krupinski, in press). The original and altered images were digitized and displayed on a high-resolution color monitor. Eye position was recorded as observers scanned the works and made aesthetic preference judgments. In such a study, the scan-path analyses are particularly informative in describing and relating changes in perceptual exploration that result from manipulations of compositional design.

\section{APPLICATIONS}

\section{Radiology}

We have been working for many years on the problem of human error in radiological interpretation. The issue that we have focused on is the early detection of lung nodules that signal the possible presence of cancer (Kundel, Nodine, \& Krupinski, 1989). This task requires searching an $X$-ray image of the chest for a small, circular, lowcontrast target. The anatomic structures of the chest image contribute pictorial clutter and noise, making the observer's search task of detecting a nodule more complicated; the nodule could easily be camouflaged by overlying structures and be missed or mistaken for a normal anatomic feature. Even though the radiological search task is carried out by an observer who is an expert at searching and interpreting radiographic images, the miss rate for early lung cancers is relatively high $(15 \%-30 \%$; see Muhm et al., 1983).

The radiological search task, like most other applied visual search tasks, draws on both global pattern recognition and local feature extraction. Usually, global pattern recognition is not successful for finding early or small lung nodules, because they are typically embedded in the anatomic context. If a nodule is not detected in the first few glances during which preattentive filtering localizes conspicuous nodules, the observer initiates a local, serially organized search strategy. The shift in emphasis from global pattern recognition to a local feature-extraction strategy to find embedded tumor targets requires the expert to scan the image by sorting and discriminating the target features from distractors and noise. Analyses of eye position and gaze duration are most effective when eyeposition data are used to describe this local featureextraction strategy of visual search.

\section{Gaze Durations and Information Processing}

Information-processing theory suggests that feature extraction consists, in part, of directing local, serial scanning fixations to regions of interest or suspicion so that image features can be sampled and tested against target criteria stored in memory. In the task of searching for small $\left(1^{\circ}\right)$, low-contrast nodules embedded in anatomic structure background, the probability of detection depends on how closely a fixation falls to a nodule target. On the basis of a study of peripheral nodule detection (Carmody et al., 1980), we have modeled the probability of detecting a nodule as a decreasing function of the eccentricity of the target from the axis of gaze (Kundel et al., 1987). This has led to probability estimates of an effective visualfield size of $2^{\circ}-3^{\circ}$ radius, which are similar to estimates observed by others (Mackworth, 1976). Detection studies like these have been used to determine the spatial thresholds in our fixation and clustering algorithm described earlier. 
With our technique of mapping eye-position data directly over displayed images, and relating gaze position and duration to specific image regions/features, we have found that gaze duration, as defined by aggregating cumulative nonconsecutive fixation clusters (rather than individual fixations), is a useful descriptor of information processing and decision making (Kundel et al., 1989). One of the main advantages of this gaze-duration analysis is that it permits us to determine how often an observer returns to a particular image location or feature, how this affects gaze-duration times, and how these gaze durations relate to the type of decision made about that image location. We assume that each eye-fixation cluster represents a decision about the presence or absence of a target within the local region of interest, as defined by the spatial extent of the fixations within that cluster. Furthermore, we assume that detection probability extends over a circular field beyond the center of the fixations that make up that cluster. To model this, we typically draw a circle with a $2.5^{\circ}$ radius around the cluster to indicate a zone of detectability. A target falling within this zone is considered to be "hit" by the fixation cluster, meaning that target detection is probable.

One method of visualizing the association of eyeposition and gaze-duration data with stimulus regions is shown in Figure 5, which presents a theoretical probability distribution for detecting a faint target embedded in a complex background, during a single fixation, as a function of displacement from the gaze axis.

To test the assumption that each eye-fixation cluster represents a decision (overt or covert), we correlate the times (gaze durations) required to process local areas of interest on the image with the observer's interpretations leading to diagnostic decisions of the image. Each of the observer's responses is coded within a signal detection framework, an approach similar to that used by picturememory researchers to infer information-processing operations by relating eye movements to verbal protocols (see Loftus, 1976, p. 501). The unit of analysis for inferring information-processing operations, gaze duration, is defined in a way almost identical to that used by Just and Carpenter (1976, 1988). Over a number of trials, the distributions of gaze durations associated with particular image features or types of decisions can be defined.

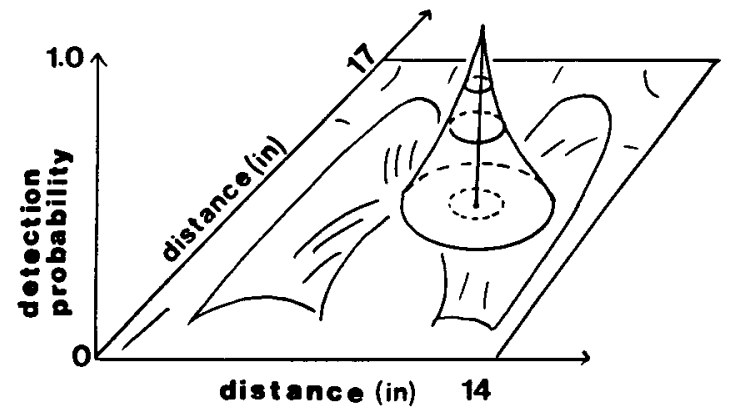

Figure 5. A theoretical probability distribution for detecting a faint target embedded in a complex background during a single fixation as a function of displacement from the gaze axis.

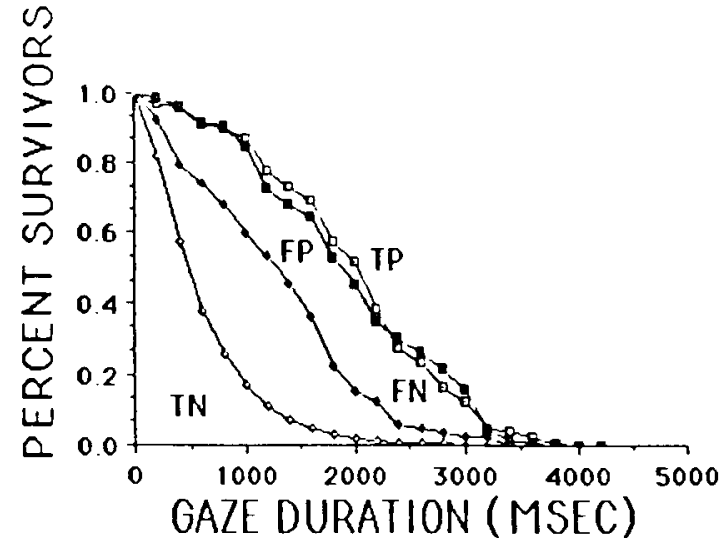

Figure 6. Survival curves generated by recording eye-position data while radiologists searched chest $X$-ray images for lung nodules. The survival curves show distributions of gaze-duration survival times for four types of decisions-true and false, positive and negative. Each decision category has a characteristic distribution of survival times that presumably reflects the differences in information load required to make a decision. Median survival time of a gaze duration was 547 msec for true-negative decisions, $1,283 \mathrm{msec}$ for false-negative decisions, 2,091 msec for false-positive decisions, and $2,291 \mathrm{msec}$ for true-positive decisions. It is significant that false-negative decisions (misses) consistently show longer survival times than true-negative decisions. Using a gaze-duration threshold of 1,000 msec filters out three times more false- than true-negative decisions, making gaze duration a useful parameter in a visual-feedback algorithm designed to improve observer performance.

\section{Survival Analysis}

We have found that the correlation of gaze duration, target location, and decision can best be described by using survival analysis (see Anderson et al., 1980). Survival analysis is useful for describing the distributions of gaze durations associated with each of the four signal detection decision categories (true, false, positive, or negative), because a statistical description based on the assumption of a Gaussian distribution is not warranted, given the Poisson-like gaze-duration distributions that we have consistently found. When survival analysis is applied to gazeduration data, the resulting curves can be thought of as describing the percentage of fixation clusters ( $y$-axis) associated with a given decision category (i.e., true-positive) that have gaze durations "surviving"' at least as long as a given time interval ( $x$-axis). Fixation clusters with a gaze duration falling within a given gaze-duration interval can be thought of as "terminating" during that interval.

Figure 6 shows the survival curves generated in a typical eye-position study of the radiological search task. Each point is based on 12 observers searching 40 images for $15 \mathrm{sec}$ each, while their eye positions were recorded. The curves for each decision category indicate that survival functions differ for positive and negative decisions. Trueand false-positive decisions are typically associated with longer gaze-duration fixation clusters, meaning that positive decisions require more in-depth visual processing, which draws out the survival curves to a greater extent than do those for true- and false-negative decisions. True- 
negative decisions are associated with the shortest gazeduration fixation clusters, which differ significantly from false-negative decisions. This indicates that misses are processed in greater depth. This finding has important implications for designing a temporal threshold algorithm to differentiate true- from false-negative decisions by visual feedback.

Given the finding that false-negative decisions (target misses) are associated with significantly longer gaze durations than are true-negative decisions, we have designed a system to feed back to the observer the locations on the image receiving gaze durations that pass a certain temporal threshold. The goal of feedback is to give the observer a chance to reevaluate all of the regions of the image that he/she has scrutinized intensely (i.e., that have elicited gaze durations exceeding $1,000 \mathrm{msec}$ ), some of which the observer may have incorrectly called targetfree when a target was indeed present. In this type of feedback experiment, it should be apparent why the precise correspondence between fixations, gaze durations, and image locations is necessary.

In the feedback experiment (Kundel, Nodine, \& Krupinski, 1990), observers searched a chest radiograph for nodules while their eye positions were recorded. The system analyzed the eye-position and gaze-duration data on line, and determined which areas (defined by the fixation clusters) of the image received prolonged gaze durations. The feedback derived from using eye-position and gazeduration data to identify potential missed nodules resulted in a $14 \%$ improvement in performance, as measured by alternative free-response receiver operating characteristic Al values (Chakraborty \& Winter, 1990), when compared with a no-feedback control condition.

\section{Art and Aesthetics}

We have also used eye-position data to determine whether altering the balance of an original composition would affect the way in which a viewer looks at a painting as revealed by the distributions of short- and longduration gazes. This is assumed to reflect differences in diversive and specific exploration (Berlyne, 1971), as well as what design areas would receive the greatest visual attention (fixation density) during perceptual analysis and judgment, which presumably reflects differences in information-processing strategies.

In a recent study by Nodine et al. (in press), fixations were statistically divided into brief glances (indicative of visual surveying associated with diversive exploration) and long gazes (indicative of visual scrutiny of features associated with specific exploration). Using statistical criteria developed by Locher and Nodine (1987), fixation clusters of 300 msec or less were designated as indicating diversive exploration and fixation clusters greater than $400 \mathrm{msec}$ were designated as indicating specific exploration. This resulted in separate nonoverlapping distributions of picture coverage for short and long gaze durations, as is shown in Figure 7.

The analysis of the breakdown of the fixation distributions indicated that, in comparison with compositions in

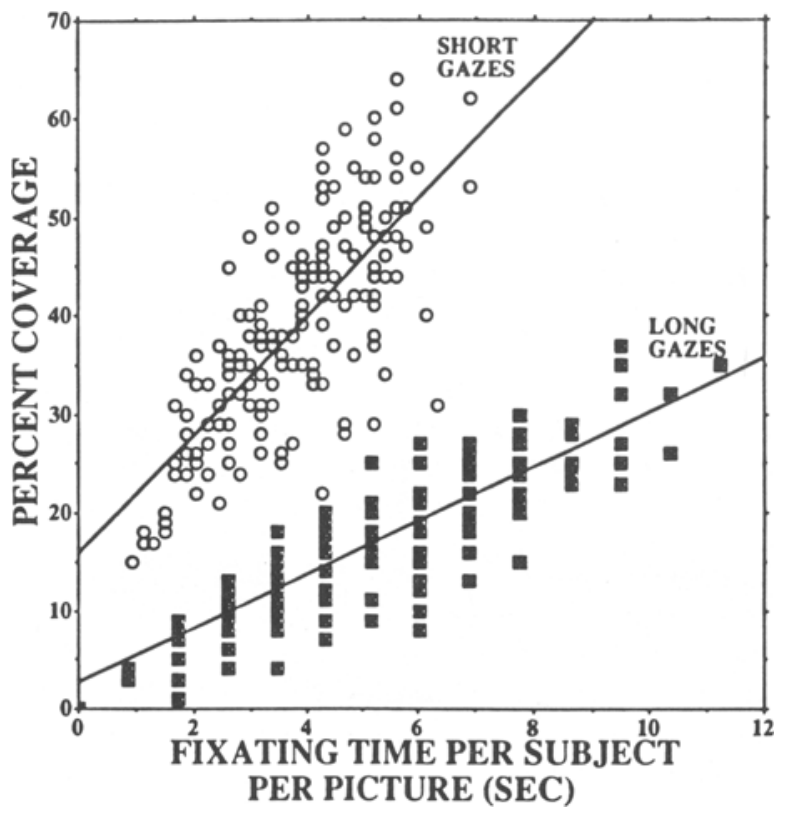

Figure 7. Percent of picture coverage by short $(<300-\mathrm{msec})$ and long ( $>400$-msec) gazes as a function of viewing time. The coverage of short gazes is consistent with the "survey" aim of diversive visual exploration, and the coverage of long gazes is consistent with the "focal analysis" aim of specific visual exploration, according to Berlyne's (1971) theory. For example, at $4.5 \mathrm{sec}$ of viewing time, short gazes cover $40 \%$ and long gazes cover only $10 \%$ of the picture.

which symmetry is a balance principle, less formally balanced compositions receive proportionally more deversive than specific exploration, and this is generally more extensive for art-trained than for untrained viewers. These differences support the notion that art training provides the viewer with a structural framework for visually exploring and judging works of art.

A second analysis was performed to determine the overall rate at which specified areas of each painting were fixated. To accomplish this area analysis, a $16 \times 16$ grid was placed over each painting, and the number of fixations (density) in each square was summed over all observers. An analysis of the compositional designs of the entire set of paintings resulted in the identification of five common design areas per painting, which are based on pictorial content: fore figure, back figure, center figure, changed figure, and background. The percentage of fixations falling on groups of grid squares containing compositional elements contributing to design balance for each pair of paintings could then be quantified.

Using this analysis, we were able to identify the density of fixations on specific areas of each painting across all observers. Areas associated with greater densities of fixations are called "hotspots" and are assumed to reflect areas of the paintings that attract attention and contribute to the aesthetic judgment of compositional design. Figure 8 illustrates a "hotspot" analysis of the fixation distribution for observers scanning Seurat's "Les Poseuses." The regions that received the greatest density of fixations 
(A)

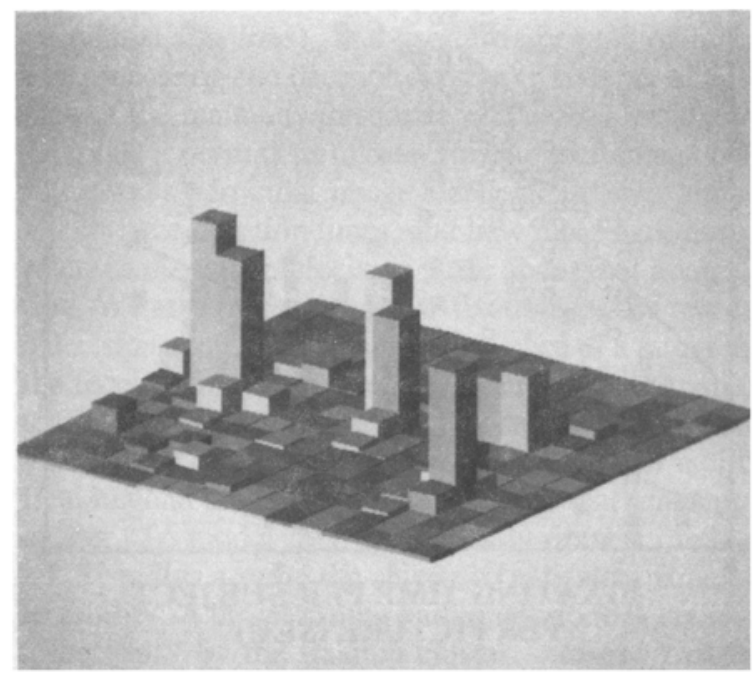

(C)

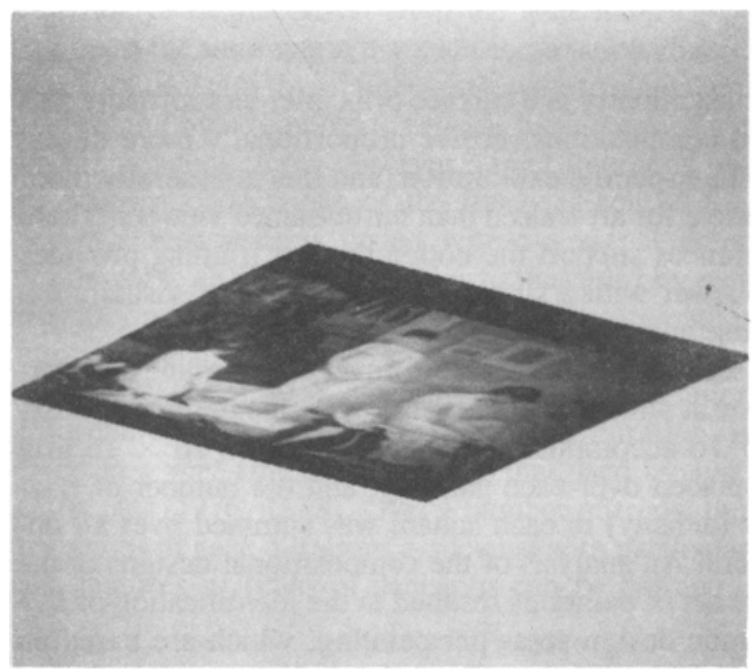

(B)

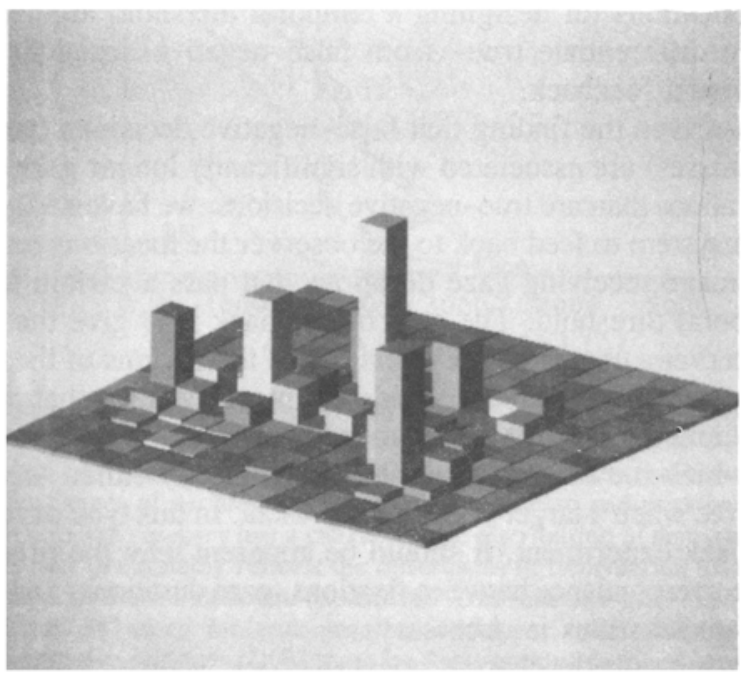

(D)

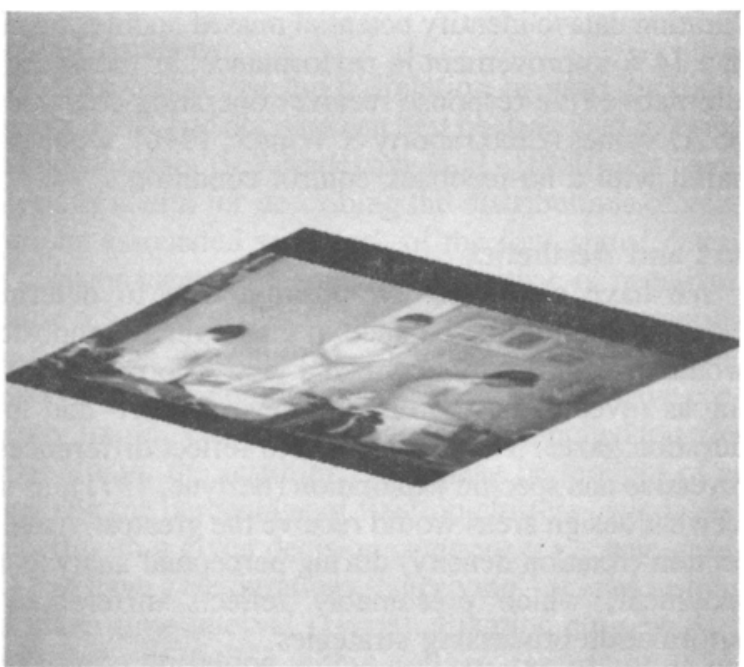

Figure 8. Three-dimensional "hot-spot" bar graphs (A and B) showing spatial distributions of fixation densities for an observer examining two versions of Seurat's painting "Les Poseuses": the original (C), and a less-balanced version (D). The greater the height of a bar in a given grid square, the greater the density of fixations associated with that region on the painting. Note the grouping of high bars on the left side of Panel A, which is associated with the detail of "La Grande Jatte" in the larger painting. The lack of detail in the same region in Panel $D$ resulted in a lower density of fixations. 
are represented by the highest grid squares. The entire display can be rotated, so it can be viewed from any perspective.

\section{CONCLUSIONS}

The emphasis of the data-collection and analysis system that we have described is on eye-position and gazeduration parameters-not eye movements. Our clustering algorithm does, however, preserve scan-path information, because we believe that it is important to capture the evolution of fixation clusters within the natural sequence of scanning a picture, particularly when the task involves the search and detection of targets. Since eye positions are presumed to correlate with the processing of local information and decisions about pictorial features in the images, our programs are designed to ensure the best possible calibration and eye-position grouping algorithms from which to test information-processing hypotheses about the way in which observers scan, search, and detect objects of interest in pictorial scenes. This has guided our development of fixation and gaze-duration analyses, which are aimed at the accurate correlation of eye-position parameters with stimulus display features.

The study of the causes of human error in radiology has led to the use of eye-position data to analyze visual information processing within the context of decision theory. Analysis of the distributions of gaze-duration data associated with clusters of fixations has been shown to reliably correlate with the type of decision (true or false, positive or negative) made about a given image feature or location. The analytic methods reported in this paper have also illustrated a number of ways in which eyeposition data can be grouped and visually represented by being superimposed on the image. This has led to a more comprehensive interpretation of visual-search tasks and other tasks involving the interpretation of pictures. Specifically, fixation, cluster, and scan-path data can be spatially remapped over a given stimulus for a twodimensional representation. Fixation density and gazeduration data can be mapped across a number of observers for a given stimulus and displayed in a three-dimensional representation. Thus, we have shown how the eye-position calibration, analysis, and the remapping of display programs can be used as tools for studying the visual processing of pictorial displays.

\section{REFERENCES}

Anderson, S., Auquier, A., Hauck, W. W., OAkes, D., VanDAELE, W., \& WEISBERG, H. I. (1980). Statistical methods for comparative studies. New York: Wiley.

Berlyne, D. E. (1971). Aesthetics and psychobiology. New York: Appleton-Century-Crofts

Carmody, D. P., Kundel, H. L., \& Nodine, C. F. (1980). Performance of a computer system for recording eye fixations using limbus reflection. Behavior Research Methods \& Instrumentation, 12, 63-66.

Chakraborty, D. P., \& Winter, L. H. L. (1990). Free-response methodology: Alternative analysis and a new observer performance experiment. Radiology, 174, 873-881.
Cornsweet, T. N. (1970). Visual perception. New York: Academic Press.

Gale, A. E., Worthington, B. S. (1983). The utility of scanning strategies in radiology. In R. Groner, C. Metz, D. Fisher, \& R. Monty (Eds.), Eye movements and psychological functions: International views (pp. 169-191). Hillsdale, NJ: Erlbaum.

GALE, A. G., Worthington, B. S. (1984). Image viewing habits and the false diagnosis. Diagnostic lmaging, 6, 130-136.

JUST, M. A., \& CARPENTER, P. A. (1976). Eye fixations and cognitive processes. Cognitive Psychology, 8, 441-480.

Just, M. A., CARPENTER, P. A. (1988). Reading and spatial cognition: Reflections from eye fixations. In G. Luer, U. Lass, \& J. ShalloHoffman (Eds.), Eye movement research: Physiological and psychological aspects (pp. 193-213). Lewiston, NY: Hogrefe.

KUNDEL, H. L., \& LAFOlletTe, P. S., JR. (1972). Visual search patterns and experience with radiological images. Radiology, 103, 523-528.

Kundel, H. L., Nodine, C. F., \& Krupinski, E. A. (1989). Searching for lung nodules: Visual dwell indicates locations of false-positive and false-negative decisions. Investigative Radiology, 24, 472-478.

Kundel, H. L., Nodine, C. F., \& Krupinsk, E. A. (1990). Computerdisplayed eye position as a visual aid to pulmonary nodule interpretation. Investigative Radiology, 25, 890-896.

Kundel, H. L., Nodine, C. F., Thickman, D., Toto, L. (1987). Searching for lung nodules: A comparison of human performance with random and systematic scanning models. Investigative Radiology, 22, 417-422.

KUNDEL, H. L., \& WRIGHT, D. J. (1969). The influence of prior knowledge on visual search strategies during the viewing of chest radiographs. Radiology, 93, 315-320.

LATIMER, C. R. (1988). Eye-movement data: Cumulative fixation time and cluster analysis. Behavior Research Methods, Instruments, \& Computers, 20, 437-470.

Lesgold, A., Rubinson, H., Feltovich, P., Glaser, R., KlopFER, D., WANG, Y. (1988). Expertise in a complex skill: Diagnosing X-ray pictures. In M. T. H. Chi, R. Glaser \& M. J. Farr (Eds.), The nature of expertise (pp. 311-342). Hillsdale, NJ: Erlbaum.

LOCHER, P. J., \& Nodine, C. F. (1974). The role of scanpaths in the recognition of random shapes. Perception \& Psychophysics, 15, 308-314.

Locher, P. J., Nodine, C. F. (1987). Symmetry catches the eye. In J. K. O'Regan \& A. Levy-Schoen (Eds.), Eye movements; From physiology to cognition (pp. 353-361). Amsterdan: Elsevier.

LofTus, G. R. (1976). A framework for a theory of picture recognition. In R. A. Monty \& J. W. Senders (Eds.), Eye movements and psychological processes (pp. 499-513). Hillsdale, NJ: Erlbaum.

MACKWORTH, N. H. (1976). Stimulus density limits the useful field of view. In R. A. Monty \& J. W. Senders (Eds.), Eye movements and psychological processes (pp. 307-321). Hillsdale, NJ: Erlbaum.

Muhm, J. R., Miller, W. E., Fontana, R. S., Sanderson, D. R., \& UHLENHOPP, M. A. (1983). Lung cancer detection during a screening program using four-month chest radiographs. Radiology, 148, 609-615.

Nodine, C. F., CARmody, D. P., \& Kundel, H. L. (1978). Searching for NINA. In J. W. Senders, D. F. Fisher, \& R. A. Monty (Eds.), Eye movements and the higher psychological functions (pp. 241-258). Hillsdale, NJ: Erlbaum.

Nodine, C. F., Locher, P. J., \& Krupinski, E. A. (in press). The role of formal art training on perception and aesthetic judgment of art compositions. Leonardo.

Noton, D., \& StaRK, L. (1971). Eye movements and visual perception. Scientific American, 224, 34-43.

Scinto, L. F. M., Barnette, B. D. (1986). An algorithm for determining clusters, pairs or singletons in eye-movement scan-path records. Behavior Research Methods, Instruments, \& Computers, 18, $41-44$.

STARK, L., Elus, S. R. (1981). Scanpaths revisited: Cognitive models direct active looking. In D. F. Fisher, R. A. Monty, \& J. W. Senders (Eds.), Eye movements: Cognition and visual perception. Hillsdale, NJ: Erlbaum.

(Manuscript received April 15, 1991; revision accepted for publication February 18, 1992.) 